\title{
Transanal Endoscopic Video Assisted Rectal Lesion Resection, New Techniques Addressing Neoplasms
}

\author{
Ali Mahmood ${ }^{1}$, Javier Nieto ${ }^{1}$, Madhu Ragupathi ${ }^{1}$, Prianka Gajula ${ }^{2}$ \\ ${ }^{1}$ Division of Minimally Invasive Surgery, Department of Surgery, \\ University of Texas at Houston Medical School, Houston, USA \\ ${ }^{2}$ Colorectal Surgical Associates, Sugar Land, USA \\ Email: StrikerMD@hotmail.com
}

Received March 13, 2012; revised April 30, 2012; accepted May 12, 2012

\begin{abstract}
Early rectal cancer beyond the reach of conventional instruments has resulted in major abdominal and pelvic operations. As visualization is compromised beyond $6-8 \mathrm{~cm}$, proximal to the anal verge, there have been several innovations and techniques to address T1 or T2 rectal cancer in the mid to upper rectum. Transanal Endscopic Microsurgery (TEM) was a technique that had garnered some success, however with expensive instrumentation along with limitations in instrument mobility, this technology was not applicable to many patients. Transanal Endoscopic Video Assisted (TEVA) rectal resection offers a cheap and readily accessible media to address early rectal cancer. Any hospital with standard laparoscopic ability is capable to offer TEVA rectal resection. We do advocate appropriate patient selection and advise that there is a learning curve with the increased requirement for technical difficulty. Once mastered, however, this surgical approach does provide yet another tool in the armamentarium of the surgeon.
\end{abstract}

Keywords: Rectal Cancer; Minimally Invasive Surgery; Endoscopic Surgery

\section{Transanal Endoscopic Video Assisted (TEVA) Rectal Lesion Excision}

Lesions in the mid to distal rectum have often required a formal oncologic resection. While this has been technically feasible, it does preclude a certain mobidity, including bleeding and nerve damage particularly in reference to the pelvic anatomy. Lesions that are not amenable to endoscopic removal must be resected, however, until recently there were not many alternatives available except for major abdominal and pelvic surgery.

Transanal Endoscopic Video Assisted (TEVA) rectal lesion excision offers a different avenue to address rectal masses. This technique offers a full thickness rectal excision without an abdominal operation or pelvic dissection. There does exist a very strict criteria of patient selection before TEVA procedures can be offered to the patient:

1) Rectal lesions must be benign or early stage adenocarcinoma not more than (uT1N0);

2) The anatomical location of the rectal lesion must not extend beyond $10 \mathrm{~cm}$ proximally or $5 \mathrm{~cm}$ distally;

3) Lesions must not encompass greater than $40 \%$ of the intraluminal rectal wall.

These criteria ensure maximal success from our experience.

*There are not any financial contributions or influences associated with this editorial.
Patients are placed in lithotomy position irrespective of the lesion location or orientation. A SILS ${ }^{\mathrm{TM}}$ (Covidien, Mansfield, MA) port is introduced into the rectum and secured in place (Figure 1). The rectum is insuflated with carbon dioxide. It is important that the patient can be paralyzed completely to prevent inadvertent valsalva maneuvers and maintain pneumo-rectum. Standard laparoscopic equipment is used along with a 30 degree optic scope. The SILS ports are offset and staggered to minimize instrument interference.

Following sustained rectal distension with carbon dioxide, the rectal lesion is identified and the outer mucosal margins are marked using electrocautery in a circumferential manner (Figure 2). Using a Harmonic Scalpel ${ }^{\mathrm{TM}}$ (Ethicon, Cincinnati, OH) the rectal mucosa is incised, to a plane where the perirectal fat is identified. This marks a full thickness rectal excision (Figure 3). We recommend starting with the proximal margin, as this is often the most difficult margin to obtain. Once the deep margin (peri-rectal fat) is identified, the harmonic scalpel is an excellent choice to use in circumferentially excising the lesion. The rectal mucosa has an abundant blood supply and using the harmonic scalpel facilitates hemostasis. A laparoscopic suction/irrigator is important to have available, particularly as there is a significant smoke "cloud" from the electrocautery and harmonic scalpel. Once the 


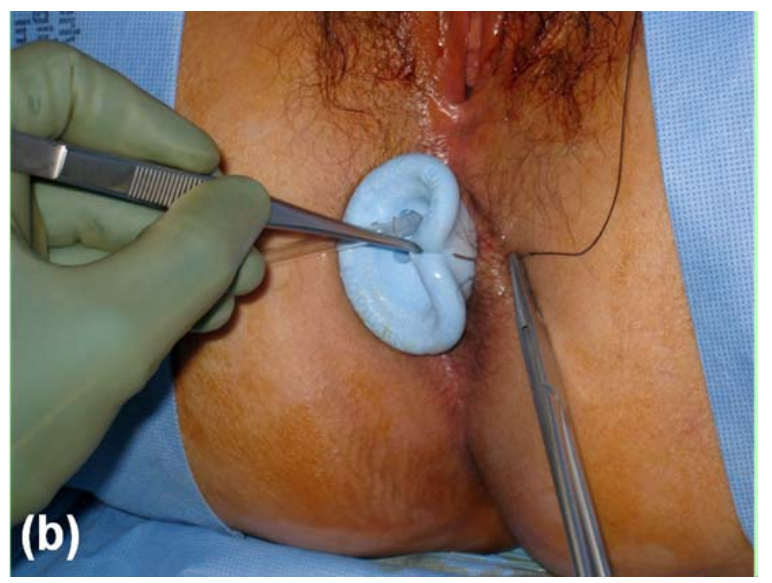

Figure 1. SILS ${ }^{\mathrm{TM}}$ port introduced into the anorectal canal and secured in place with 2 - 0 silk suture.

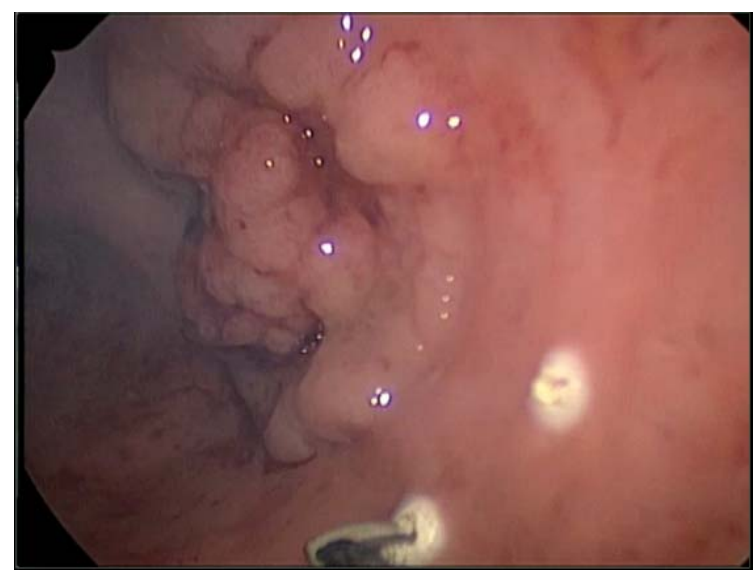

Figure 2. The circumferential margins of the lesion are marked using electrocautery.

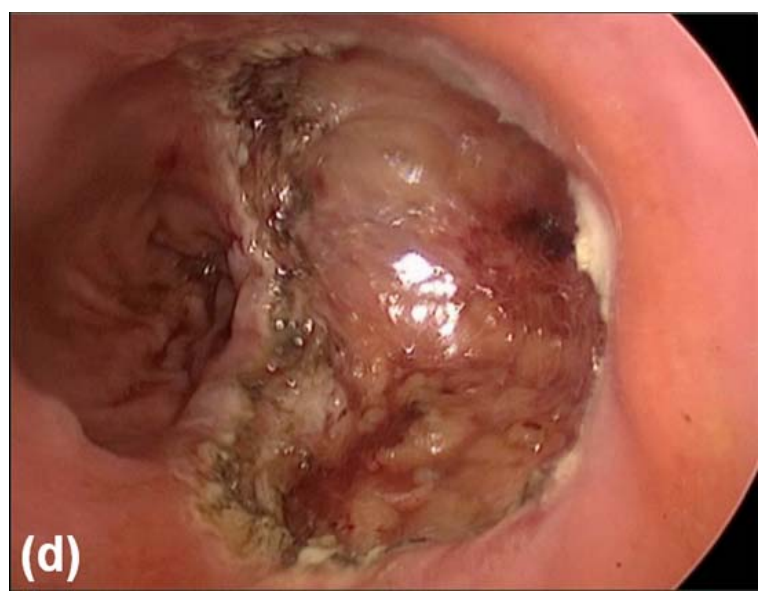

Figure 3.The rectal defect following excision. Note the perirectal fat depicting a full thickness excision.

lesion is completely excised, the port is removed to facilitate extraction, however great care is used to ensure that the orientation of the specimen is maintained. The specimen is marked and orientated for pathology.
The rectal defect can be closed using the SILS port. Standard laparoscopic suture technique is technically feasible yet meticulous. The authors have used the V-LOC (Covidien, Mansfield, MA) closure device to aid in the closure of the defect (Figure 4). Using a laparoscopic needle driver and a Maryland grasper, the V-LOC suture is passed into the rectal canal. The needle is not loaded onto the needle driver until it is inside the rectal canal. The aperture of the Covidean SILS ports do not allow a loaded needle to pass through and it has the danger of getting stuck inside the port. The suture is grasped just proximal to the needle and passed into the canal. Once inside the rectum, the needle driver and Maryland grasper are used to close the defect. We recommend the Maryland grasper because of its fine tip and ability to maneuver the needle and grasp the rectal mucosa in a precise, yet delicate manner. It is better to start at the most proximal apex of the rectal defect and work in a distal direction to visualize the entire defect and suture it closed.

Wile TEVA rectal lesion resection offers excellent visualization and approach to mid rectal lesions we underscore the importance of pre-operative patient selection. Lesions T2 or advanced should undergo a formal oncologic resection with total mesorectal excision (TME), particularly with their associated high rate of recurrence and lymph node invlolvement [1,2]. Although it has been reported that T2 lesions can be locally resected with adjuvant chemotherapy, and yield favorable results, we prefer formal oncologic resection with TME for these cancers, when feasible [3-5]. The applicability of this newer technology and technique is aimed primarily at early rectal cancer lesions that are beyond the reach of a standard transanal approach, however are amenable to local excision. Prior to TEVA, early rectal cancer that was not able to be removed via the transanal approach required a major pelvic dissection. TEVA resection offers a method to remove these mid rectal lesions with most patients discharged home the same day of the procedure. It is yet

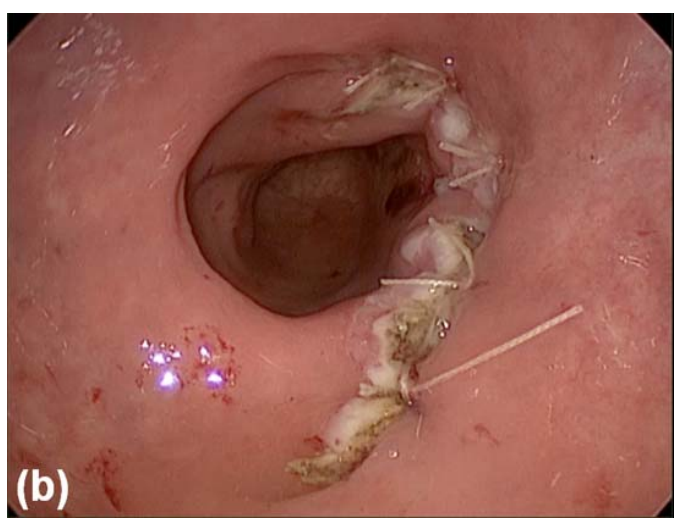

Figure 4. The rectal defect closed using interrupted suturing technique. 
one more tool in the armamentarium of the surgeon addressing rectal cancer.

\section{REFERENCES}

[1] J. E. Krook, C. G. Moertel, L. L. Gunderson, et al., "Effective Surgical Adjuvant Therapy for High Risk Rectal Carcinoma,” New England Journal of Medicine, Vol. 310, 1991, pp. 737-743.

[2] J. T. Brodsky, G. K. Richard, A. M. Cohen and B. D. Minsky, "Variables Correlated with the Risk of Lymph Node Metastasis in Early Rectal Cancer,” Cancer, Vol. 69, No. 2, 1992, pp. 322-326. doi:10.1002/1097-0142(19920115)69:2<322::AID-CNCR
2820690208>3.0.CO;2-B

[3] G. D. Steele Jr., J. E. Herndon, R. Bleday, et al., "Sphincter-Sparing Treatment for Distal Rectal Adenocarcinoma,” Annals of Surgical Oncology, Vol. 6, No. 5, 1999, pp. 433-441.

[4] R. Bleday, E. Breen, J. M. Jessum, et al., "Prospective Evaluation of Local Excision for Small Rectal Cancers," Diseases of the Colon \& Rectum, Vol. 40, No. 4, 1997, pp. 388-392. doi:10.1007/BF02258381

[5] D. M. Ota, "MD Anderson Cancer Center experience with Local Excision and Multimodality Therapy for Rectal Cancer," Surgical Oncology Clinics of North America, Vol. 1, No. 1, 1992, pp. 147-152. 